\title{
Isolation and characterization of Saccharomyces cerevisiae genes differentially expressed under different growth conditions
}

\author{
Wen-yan Wang, Teppei Nishikawa, and Katsumi Isono* \\ Division of Bioscience, Postgraduate School of Science and Technology, \\ Kobe University, Kobe 657, Japan
}

(Received July 24, 1997; Accepted August 22, 1997)

\begin{abstract}
The budding yeast Saccharomyces cerevisiae, like many other microorganisms, responds to nutrient starvation by arresting growth and entering into a non-proliferating stationary phase. Studies on the response of $S$. cerevisiae cells to growth arrest might provide further insight into the nonproliferative states of cells in multi-cellular eukaryotic organisms. Changes might occur at the transcription, translation, and post-translational levels in cells upon entry into the stationary phase. To search for the genes differentially expressed in yeast cells during different growth phases, we have performed systematic Northern hybridization experiments using probes prepared for a large number of genes/ORFs. We have thus isolated and characterized 42 cDNA clones containing genes hyper-expressed in the post-diauxic phase. Some of them have already been characterized, and many others show similarity to known yeast genes or genes of other organisms. However, eleven of them were found to be unrelated to any known genes. We have characterized some of these genes as described below. Also, a possible cis-element for transcriptional regulation was identified.
\end{abstract}

Key Words___ growth conditions; likely cis-elements; Northern hybridization; post-diauxic phase; Saccharomyces cerevisiae genes

In nature, the cessation of growth of living organisms is often brought on by poor nutrient environments, which is one of the most commonly encountered stresses. Environmental changes subsequently affect various biochemical reactions, often disturbing the balanced distribution of metabolites within cells. In most instances, living cells show a rapid molecular response to overcome such adverse environmental conditions. When cells of unicellular microorganisms encounter such unfavorable nutrient conditions, they ultimately enter into a stationary phase. Cells in the stationary phase are physiologically, biochemically, and morphologically different from cells growing exponentially. Studies with Escherichia coli (Kolter et al., 1993; Weiner and Model, 1994) and yeast (Ireland et al., 1994; Mager and Ferreira, 1993; Werner-Washburne et al., 1993, 1996) indicate that entry into the stationary phase is a complex, highly regulated process that activates a program for long survival without added nutrients and in the absence of cell division. The similarities of eukaryotic and prokaryotic microorganisms

\footnotetext{
* Address reprint requests to: Dr. Katsumi Isono, Division of Bioscience, Postgraduate School of Science and Technology, Kobe University, Rokkodai, Kobe 657, Japan.
}

in their responses to nutrient limitation suggest that such responses are based on evolutionary conserved genetic mechanisms. It has been proposed that starvation causes yeast cells to enter into the $G_{0}$ phase, in which they remain viable for prolonged periods of time (Werner-Washburne et al., 1996). The molecular tractability of Saccharomyces cerevisiae and the universality of the cellular responses to nutrient limitation support the idea that an analysis of yeast cells will provide useful information with respect to general cellular responses to nutrient limitations and the subsequent physiological changes.

S. cerevisiae is one of the simplest and best studied eukaryotes. It has a small genome $(12.5 \mathrm{Mb})$ separated into 16 chromosomes and contains little repetitive DNA and few introns. The genome of $S$. cerevisiae has been estimated to contain approximately 6,000 genes, of which about 2,500 have been biologically characterized till now. The remaining genes include some 2,000 genes that are considered to be $S$. cerevisiae-specific ("orphan genes"). The entire genomic nucleotide sequence has already been determined and made available to the public through the DDBJ/EMBL/GenBank nucleotide sequence database. This will speed up yeast genetic studies to a 
great extent. To understand the process of cells entering into non-proliferative states in greater details, we have focused our attention to the analysis of yeast genes that are differently expressed in logarithmically growing cells and in cells after diauxic shift (i.e., a shift from fermentation to respiration). For this purpose, cDNA libraries were constructed, and genes, the transcription of which was enhanced in cells in a post-diauxic phase relative to those in cells growing exponentially, have been identified by differential screening and Northern hybridization. Positive clones were sequenced and their genes were identified and located on the yeast genomic data. The results indicate that genes in a variety of metabolic reactions change their expression levels in the post-diauxic phase, and that some of the genes thus identified are also hyper-expressed under other stress responses such as heat shock.

\section{Materials and Methods}

Bacterial strain and plasmids. The E. coli strain used for the construction of the CDNA library was XL-1 Blue recA1 lac endA1 gyrA96 thi hsdR17 supE44 relA1 $\Delta\left(\right.$ lac-proAB), $\mathrm{F}^{\prime}$ (proAB ${ }^{+}$laq ${ }^{q}$ lacZ $\Delta M 15$ Tn10). It was grown in LT medium ( $1 \%$ bacto-tryptone, $0.5 \%$ yeast extract, $0.5 \% \mathrm{NaCl}, 0.002 \%$ thymidine) at $37^{\circ} \mathrm{C}$. Plasmids pUC118 and pUC119 were used for cloning and subcloning.

Yeast cultures. The yeast strain used in these experiments was DC5 a, leu2-3 leu2-112 his3 can1. It was routinely grown in YPD medium (1\% yeast extract, $2 \%$ peptone, $2 \%$ glucose) at $28^{\circ} \mathrm{C}$. For the preparation of total RNA, cells after culturing overnight were diluted by fresh YPD medium and harvested when the cell density reached either $\mathrm{OD}_{650}$ of 2.0 (logphase) or $\mathrm{OD}_{650}$ of 15-20 (post-diauxic phase).

Preparation and screening of CDNA library. Total RNA was prepared essentially as described by Sprague et al. (1983) from cells in post-diauxic phase as defined above. For the preparation of $\operatorname{poly}(A)^{+}$ RNA, the total RNA was passed through an oligo (dT) cellulose column (BRL) either once or twice. Five micrograms of the resultant poly $(A)^{+}$RNA were used to prepare a cDNA library using a TimeSaver cDNA synthesis kit (Pharmacia). All procedures were performed according to the supplier's protocol. The insert in each clone of the cDNA library was then amplified by PCR with the primers located on both sides of the multicloning site. Blotting membrane (Pall Biodyne, $\phi=$ $1.2 \mu \mathrm{m})$ was prepared in duplicate as described by Ausubel et al. (1987). The log- and post-diauxic phase cDNA preparations were labeled using random primers, and $\left[{ }^{32} \mathrm{P}\right]$-dATP and cDNAs showing stronger hybridization with the latter were selected for further analysis.

Northern blot analysis. The standard methods of Sambrook et al. (1989) were mostly followed. Two micrograms of poly $\left(\mathrm{A}^{+}\right)$RNA from log- or post-diauxic phase cells were electrophoresed in each lane of $1 \%$ agarose gel (LO3, Takara Shuzo) under denaturing conditions $(0.66 \mathrm{M}$ formamide in $20 \mathrm{~mm}$ MOPS, $\mathrm{pH} 7.0$, $5 \mathrm{~mm} \mathrm{Na}$-acetate and $1 \mathrm{~mm}$ EDTA) and transferred onto a nylon membrane (Pall Biodyne, $\phi=0.2 \mu \mathrm{m}$ ). DNA probes were prepared from respective plasmids by digestion with an appropriate restriction endonuclease and electrophoresis on a mini-gel $(5.2 \times 6.0 \mathrm{~cm})$ of $1 \%$ SeaKem GTG agarose. The desired DNA bands were recovered from the gel using a Jet-sorb DNA isolation kit (Genomed $\mathrm{GmbH})$ and labelled with $\left[{ }^{32} \mathrm{P}\right]-$ dATP.

Gene disruption. Yeast genes were disrupted by introducing a Tn3 transposon and selecting for $\mathrm{His}^{+}$ using $E$. coli strains RDP146 $\mathrm{F}^{-}$recA1 $\triangle$ (lac-proAB) rpsE and NS2114Sm $\mathrm{F}^{-}$recA1 $\lambda$-cre rpsL, and plasmids pHSS6 $\left(\mathrm{Km}^{r}\right)$, pLB101 $\left(\mathrm{Cm}^{r}\right.$, tnpA) and pOX38:: $\mathrm{mTn} 3\left(\mathrm{HIS}^{+}\right)$as described by Seifert et al. (1986). The genomic DNA of the disruptant was then prepared, digested with appropriate restriction enzymes and electrophoresed. They were blotted onto a nylon membrane (Pall Biodyne, $\phi=1.2 \mu \mathrm{m}$ ) and hybridized with each of the DNA probes labelled with $\left[{ }^{32} P\right]-d A T P$ (Ausubel et al., 1987).

Nucleotide sequencing. Plasmid DNA was prepared according to the sequencing template preparation method of PE Applied Biosystems. Nucleotide sequencing was carried out using a PE Applied Biosystems Prism-310 capillary DNA sequencer.

\section{Results and Discussion}

Identification of genes hyper-expressed in the post-diauxic phase

A cDNA library was constructed from cells in the post-diauxic phase as described in MATERIALS AND METHODS, and was screened by hybridizing the PCRamplified cDNA fragments of this library with the cDNA probes prepared from cells in the log- and postdiauxic phases. The PCR products were spotted onto a nylon membrane in duplicate and were subsequently hybridized with the probes. The results (not shown) indicate that some of the clones were expressed distinctly more in post-diauxic phase cells than in log-phase cells, and consequently, they were selected as candidates of clones containing genes responding to the post-diauxic phase. The selected clones were then subjected to Northern hybridization using the ACT1 gene as a control. Out of approximately 850 cDNA clones initially screened, 105 were confirmed to contain transcripts which were more 
abundant in post-diauxic phase cells than in log-phase cells. By comparing the Northern hybridization patterns, cDNA clones were categorized into three groups,,+++++ and + (Table 1$)$. Figure 1 depicts the results of about half of the clones analyzed in this way.

It should be noted that the quantity of the products of Northern hybridization is influenced not only by the transcriptional activity of a gene but also by the stability of the mRNA synthesized. Therefore, it is generally

Table 1. Yeast genes hyper-expressed in the stationary phase.

\begin{tabular}{|c|c|c|c|c|}
\hline Chromosome & Clone & Northern & ORF & Gene \\
\hline 1 & V-69 & ++ & YAL062w & $\mathrm{GDH3}$ \\
\hline II & II-20 & ++ & YBL064c & thiol-specific antioxidant \\
\hline II & C-15 & +++ & YBR072w & HSP26 \\
\hline II & $1-47$ & + & YBR118w & TEF2 \\
\hline II & IV-7 & + & YBR151w & unknown \\
\hline III & VII-92 & ++ & YCL064c & CHA1 \\
\hline III & VI-87 & ++ & YCL040w & GLK1 \\
\hline IV & $11-72$ & ++ & YDL244w & homolog of $T H I 5$ \\
\hline IV & IX-69 & +++ & YDR049w & unknown \\
\hline IV & $1-40$ & +++ & YDR171w & HSP42 \\
\hline IV & IX-35 & +++ & YDR244w & PAS10 \\
\hline IV & $\mathrm{I}-60$ & +++ & YDR382w & RPLA4 \\
\hline V & VII-60 & + & YER021w & SUN2 \\
\hline V & V-56 & ++ & YER150w & unknown \\
\hline VI & $11-72$ & ++ & YFL058w & THI5 \\
\hline VI & VII-80 & + & YFL031w & $H A C 1$ \\
\hline VI & III-7 & +++ & YFL014w & HSP12 \\
\hline VII & $V-40$ & + & YGL048c & SUG1 \\
\hline VII & $11-29$ & ++ & YGR136w & unknown \\
\hline VII & $\mathrm{I}-110$ & +++ & YGR192c & $T D H 3$ \\
\hline IX & II-52 & + & YIL022w & TIM44 \\
\hline$x$ & $1-110$ & +++ & YJL052w & $T D H 1$ \\
\hline$x$ & $\mathrm{I}-110$ & +++ & YJR009c & $T D H 2$ \\
\hline$X I$ & II-56 & + & YKL142w & MRP8 \\
\hline$X I$ & II-26 & ++ & YKL109w & HAP4 \\
\hline XI & $1-46$ & ++ & YKL103c & $L A P 4$ \\
\hline$X I$ & II-54 & ++ & YKL060c & $F B A 1$ \\
\hline$X I$ & II-57 & + & YKL038w & $R G T 1$ \\
\hline XI & S-47 & + & YKR011c & unknown \\
\hline$X I$ & $\mathrm{I}-51$ & ++ & YKR046c & unknown \\
\hline$X I$ & $V-11$ & +++ & YKR097w & PCK1 \\
\hline XII & $1-42$ & + & YLR249w & YEF3 \\
\hline XII & II-59 & ++ & YLR324w & unknown \\
\hline XII & C-4 & +++ & YLR377c & $F B P 1$ \\
\hline XIII & S-112 & + & YMR267w & PPA2 \\
\hline XIII & C-10 & + & YMR297w & PRC1 \\
\hline XIII & VIII-8 & ++ & YMR302c & PRP12 \\
\hline XIV & $11-72$ & ++ & YNL332w & THI12 \\
\hline XIV & $\mid-111$ & +++ & YNL160w & $Y G P 1$ \\
\hline XIV & C-8 & +++ & YNL055c & POR1 \\
\hline XIV & S-13 & +++ & YNR034w & SOL1 \\
\hline$X V$ & IX-55 & +++ & YOL052c-a & DDR2 \\
\hline$x V$ & $1-58$ & + & YOR008c & SLG1 \\
\hline$X \mathrm{VI}$ & $\mathrm{I}-120$ & + & YPL061w & $A L D 6$ \\
\hline$X V I$ & VI-94 & + & YPL048w & CAM1 \\
\hline XVI & $1-79$ & +++ & YPR032w & unknown \\
\hline
\end{tabular}

The 'Northern' column lists scores of Northern hybridization experiments: +++ indicates most distinct differences between hybridization with the post-diauxic phase mRNA and the log-phase mRNA, ++ considerable differences, and + weak but reproducible differences (see Fig. 1 for examples). The names listed in the 'Gene' column are those adopted in the MIPS database (http://speedy.mips.biochem. mpg.de/mips/yeast): 'unknown' indicates that there is only similarity to the genes or putative genes of other organisms in the nucleotide and/or protein sequence databases, and the genes have not been analyzed in yeast. ORFs YBL064c, YDL244w and YOR008c should also be classified into this category, since they have not been experimentally characterized in yeast. Clones II-72 and I-110 contain multi-copy genes (see text). 

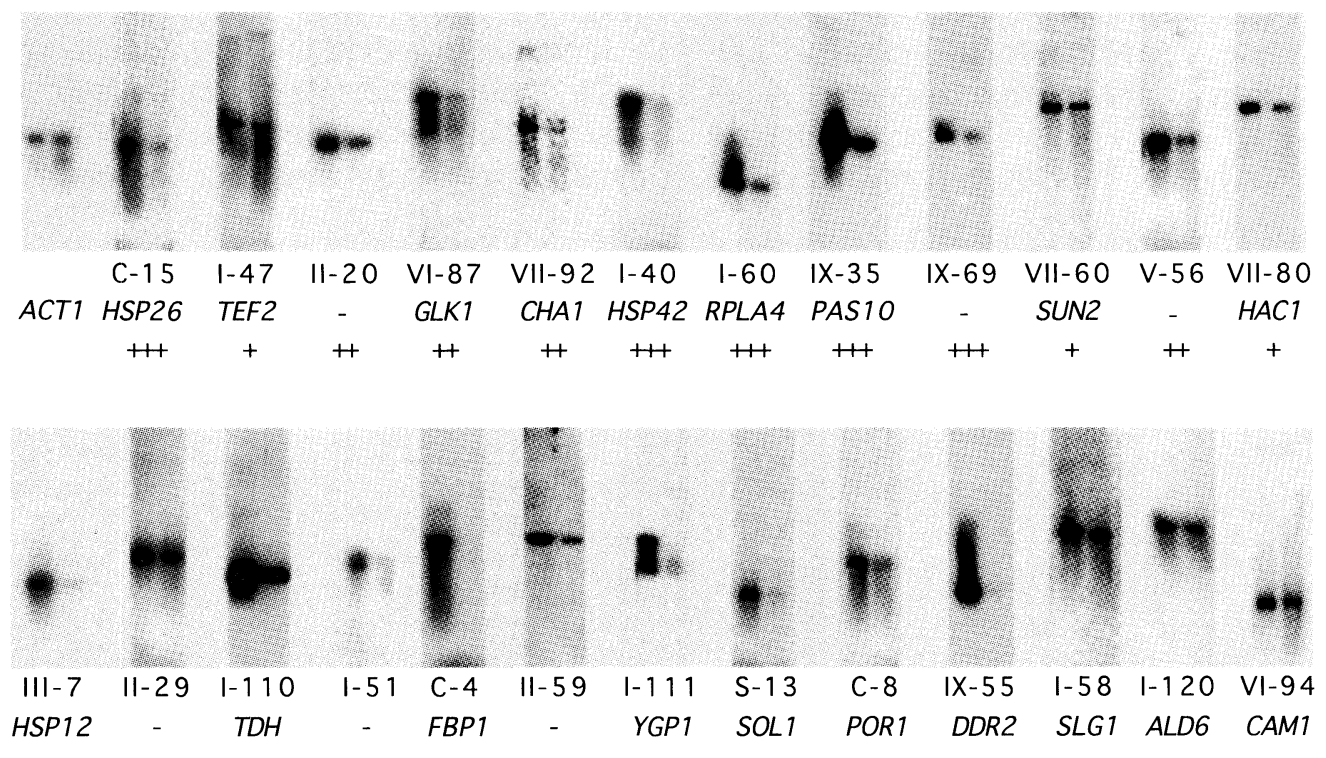

Fig. 1. Northern blot analysis of the genes hyper-expressed in the post-diauxic phase.

Cells cultured in YPD medium at $28-30^{\circ} \mathrm{C}$ were harvested at $O D_{650}$ of 2.0 (log-phase) or 15-20 (post-diauxic phase), and total RNAs were extracted and mRNA concentrated as described in MATERIALS AND METHODS. Two micrograms/lane of post-diauxic phase (left lane) or log-phase (right lane) mRNA were analyzed by Northern blot hybridization with each of the cDNA clones. An ACT1 gene probe was used as the hybridization control. The genes identified by nucleotide sequencing are listed under the clone names along with the results of their relative Northern hybridization differences (i.e.,,+++++ and + ) as shown in Table 1.

necessary to analyze both the synthesis and degradation rates of each mRNA under different growth conditions. However, the aim of our work presented in this paper was to screen as many genes as possible, the transcripts of which differ in amount under different growth conditions. We did not estimate the synthesis rate nor the stability of the mRNA. Consequently, the term 'transcriptional activity' is only loosely used throughout this paper and should therefore be taken to indicate the combined effects of the synthesis and degradation of each $\mathrm{mRNA}$.

Each of the 105 cDNA clones thus identified to harbor a post-diauxic phase responding gene was then subjected to nucleotide sequencing as described in Materials AND Methods. Many clones were found to contain identical genes redundantly, and consequently, the 105 cDNA clones analyzed in this way were sorted into a total of 42 clones as summarized in Table 1. By mapping the genes contained in them, it was found that they were distributed on almost all chromosomes as indicated. The functions of many of the genes were either already known or inferred based on their similarity to other yeast genes or to the genes of other organisms. However, eleven of the clones contained genes whose functions remained unknown: clones I-51, II-29, II-59, V-56 and IX-69 are five such examples, the expression patterns of which are shown in Fig. 1. The increase in the amount of transcripts in the post-diauxic phase of some of the clones analyzed was not so pronounced in comparison with others, such as that for clones IX-35, C-4, S13 and IX-55. Nevertheless, differences in the cells of the log- and post-diauxic phases were reproducibly observed.

Previous analyses showed that mutations in some genes which are hyper-expressed in the post-diauxic and stationary phases affect various cellular processes such as signal transduction, synthesis, N-terminal acetylation and secretion of various proteins, membrane biosynthesis, cell polarity, thermo-tolerance, etc. (Choder, 1991; Drebot et al., 1990; Finley et al., 1987; Valentin et al., 1987; Werner-Washburne et al., 1989). Moreover, the stored carbohydrates found to accumulate in the cytoplasm and lipid vesicles became increasingly abundant in the cytoplasm as cells enter the stationary phase (Vallari et al., 1992). The molecular mechanisms responsible for the entry into and maintenance of the stationary phase in $S$. cerevisiae, however, are not well established yet. It was proposed that cells in the stationary phase differ from logarithmically growing cells by the folded genomic DNA structure (Piñon, 1987). Such a folded DNA structure might influence, at least partly, the activation of certain genes in the stationary phase.

Two sets of genes that we have identified in this work as being hyper-expressed in post-diauxic phase 
cells are known to exist in triplicate (Table 1): one corresponded to genes termed $T D H 1, T D H 2$ and $T D H 3$, all of which were shown be active from individual mutational analysis (McAlister and Holland, 1985), while the others corresponded to two genes which have been termed THI5 and THI12. It will be interesting to investigate further whether or not the disruption of one, two or all three of them affects cell entry into the stationary phase.

We noted that many of the genes listed in Table 1 as being hyper-expressed in the post-diauxic phase are related to carbohydrate metabolism. GDH3, GLK1, TDH1-3, FBA1, and PCK1 are several of such examples. The PCK1 gene, for example, encodes phosphoenolpyruvate carboxykinase, a key enzyme in gluconeogenesis which is sensitive to glucose and whose intracellular localization is known to vary in different mammals. Its intracellular location in yeast cells has not been analyzed in detail however. In addition, several genes such as TIM44 and MRP8, whose products are related to mitochondrial function, are included in Table 1. Similarly, genes such as HSP12, HSP26 and HSP42, which are known to be heat shock-responsive, were found to be hyper-expressed in the post-diauxic phase. However, the data in Table 1 are clearly incomplete in that genes SUG1, TIM44 and HAP4, the products of which are subunits of multi-component complexes, namely the 265 proteasome, mitochondrial innermembrane receptor and CCAAT-binding factor, respectively, are included, whereas genes encoding other components of the corresponding complexes are not.

Among the genes identified in this work, those whose expression level showed the greatest difference are mostly either heat-shock protein genes or genes involved in the carbohydrate metabolism described above. Genes such as TEF2, YEF3 and SUN2, which are involved in protein synthesis and degradation, showed less significant differences. Similarly, genes such as RGT1, PPA2, HAC1 and MRP8, which encode transcriptional factors or mitochondrial proteins, also showed less significant differences. These observations might serve as clues to elucidate the functions of 'unknown' genes.

\section{Response of the identified genes to other conditions}

Previous studies showed that many stress-responsive genes also respond to other stress conditions (Werner-Washburne et al., 1993). To investigate whether or not the genes we have identified would change their transcription levels in response to other stress conditions, they were subjected to analysis by Northern hybridization with RNAs prepared from cells grown under different conditions (i.e., cells in log phase, post-diauxic phase, stationary phase, after ex-

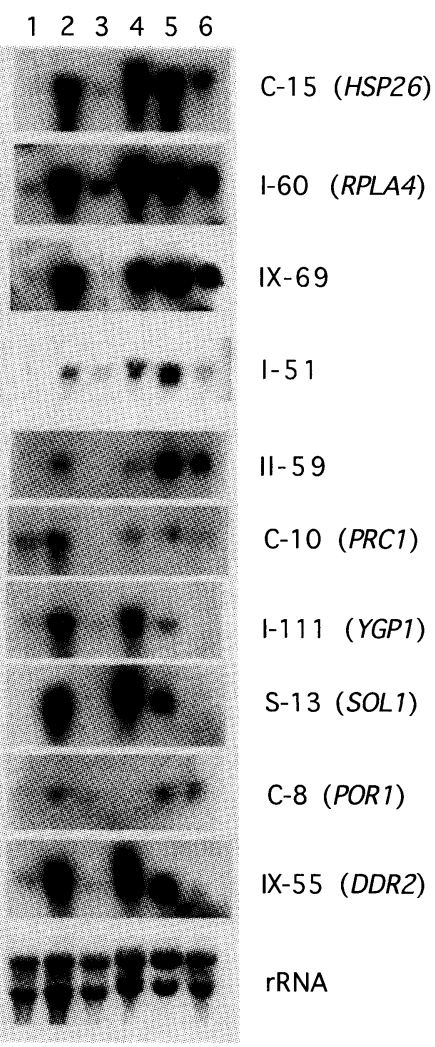

Fig. 2. Response patterns of the identified genes to other growth conditions.

Expression of the gene in each clone was examined by Northern blot analysis against the mRNA prepared from cells in the log phase (1); in post-diauxic phase (2); after heat-shock treatment (i.e., after shift of a log-phase culture from $28^{\circ} \mathrm{C}$ to $37^{\circ} \mathrm{C}$, and incubation continued for a further $1.5 \mathrm{~h}$ ) (3); in artificial high density (i.e., after concentration of a log-phase culture to make an $\mathrm{OD}_{650}$ of 20 , and incubation continued for a further $0.5 \mathrm{~h}$ ) (4); in YPG medium (5); and in the stationary phase (after 7 days) (6). Total RNAs were extracted from the cells and $7 \mu \mathrm{g}$ RNA/lane were used. Ethidiumbromide staining of rRNA is shown at the bottom. The genes identified by nucleotide sequencing are listed in parentheses.

posure to heat shock $\left(37^{\circ} \mathrm{C}\right)$, and grown in $\mathrm{YPG}$ medium). It was found that many of the genes analyzed were also hyper-expressed to varying degrees under other stress conditions, as shown in Fig. 2. Based on the response patterns to the stress conditions analyzed, the cDNA clones could be classified into 3 groups. Group 1 contains clones C-15, I-60, IX69 and I-51: the genes in this group were strongly induced under all conditions employed. Group 2 contains clones II-59, C10 and C-8: they were considerably induced in the post-diauxic and stationary phases and upon glucose deficiency, but not so much in high cell density. In addition, their responses were generally much less pronounced. Group 3 contains clones I111, S-13 and IX-55: these genes were strongly induced in the post-diauxic phase and in high cell density, but responded negligibly in the stationary phase. 
For most of the genes analyzed, their responses could be interpreted as reasonable from their known functions. However, some of them were unexpected. Clone 1-60 contains the gene termed RPLA4, which encodes ribosomal protein $L 45$. It is interesting to note that this gene is hyper-expressed under various stress conditions, since there are as many as 80 ribosomal protein genes in $S$. cerevisiae, and moreover, many of them are in duplicate. Whether or not there are other ribosomal protein genes that are also hyper-expressed under these conditions remains to be analyzed further.

As can be seen in Fig. 2, the genes we have identified were transcribed more pronouncedly at or immediately after the diauxic shift and became less active upon entry into the stationary phase. This was particularly the case with the genes of group 3 described above. In contrast, three of the eleven newly identified genes, namely IX-69, I-51 and II-59, are hyper-expressed in the stationary phase as well. Therefore, they might be more directly involved in a cell's entry into the stationary phase. Furthermore, all of the genes analyzed except for C-8 (POR1) were induced, albeit to varying degrees, when log-phase cells were concentrated. In the case of higher eukaryotic cells, it is known that a cell-to-cell interaction, and hence, cellular concentration is important for growth. Our results indicate the possibility that yeast cells also have the ability to sense and react to cell density.

\section{Disruption analysis of three functionally unknown genes}

Clones I-51, I-79 and II-29, each of which is derived from a gene with unknown functions, were analyzed further after disruption of the gene in question using the shuttle mutagenesis method (Seifert et al., 1986) followed by Southern hybridization with haploid genomic DNA. Clone 1-51 was classified as ++ in the Northern hybridization and was found to be derived from YKR046c, which is located on chromosome XI. Although its protein product has not been characterized yet, at least our results have established that it is certainly an active gene whose expression is distinctly enhanced after the diauxic shift, since its mRNA was detected as shown in Figs. 1 and 2. Clone II-29 was also classified as ++ in the Northern hybridization experiments. Its gene is located on chromosome VII under the name of YGR136w, whose product shows a weak similarity to the chicken growth factor receptorbinding protein termed GRB2. Clone I-79 was classified as +++ . It corresponds to YPR032w, located on chromosome XVI. It shows strong similarity to another hypothetical yeast protein gene termed YBL106c.

The disruption of these three genes was conducted, and the resultant disruptants were isolated and cul- tured under different conditions: namely, at $30^{\circ} \mathrm{C}$ or $37^{\circ} \mathrm{C}$ in YPD, YPD containing $1 / 4$ of glucose, YPD containing $0.7 \mathrm{M} \mathrm{NaCl}$ or $Y P D$ containing $0.2 \mathrm{M} \mathrm{LiCl}$. No anomalies were observed with any of the disruptants analyzed under these conditions however.

Search for likely cis-elements in the sequence upstream of the stationary phase-responsive genes

Previously, a search for regulatory elements for the heat-shock protein (HSP) genes was performed (Craig et al., 1993). HSP genes contain one or more heat-shock response element (HSE), which is a binding site for the heat-shock transcription factor (HSF). A regulatory element responsible for a variety of stress conditions was initially identified as an HSF-independent heat-shock element in the promoters of CTT1 (Wieser et al., 1991) and DDR2 (Kobayashi and McEntee, 1990, 1993). These elements, containing the consensus core sequence AGGGG, were designated stress response elements (STRE), which seem responsible for various forms of stresses (Marchler et al., 1993; Ruis and Schüller, 1995; Schmitt and McEntee, 1996). Recently, it has become clear that transcriptional regulation involves sequence-specific DNAbinding proteins, and a large number of such proteins have been identified in various organisms (Dhawale and Lane, 1993). The common cis-element existing in the untranslated region upstream was used to predict the functions of the new genes on chromosome III (Fondrat and Kalogeropoulos, 1994). Identification and characterization of functionally related cis-regulatory elements may contribute to the elucidation of the functions of hitherto unknown genes that have been predicted from genomic nucleotide sequencing.

Bearing this in mind, we then examined the occurrence of oligonucleotide cis-elements common to the sequence upstream of most, if not all of the genes that were hyper-expressed in the post-diauxic phase. For this purpose, the region $500 \mathrm{bp}$ upstream of each gene/ORF was extracted from the yeast genomic nucleotide sequence data and all permutations of tetra-, penta- and hexa-nucleotide sequences were searched for in the genomic nucleotide sequence data and scored. For the sake of simplicity, we only searched for consecutive oligo-nucleotide sequences of $100 \%$ match as candidates for likely cis-elements that should be experimentally analyzed further in the future.

The STRE element was found to be common in the stretch upstream of the genes we have identified (Table 2). However, 22 out of 42 genes (52\%) did not have any STRE element in either orientation, 16 of which were found to contain instead at least one copy of a related penta-nucleotide, AAAGG (or CCTTT). This putative cis-element was found to occur also in 
Table 2. Occurrence of penta-nucleotides upstream the stationary phase responsive genes.

\begin{tabular}{|c|c|c|c|c|}
\hline ORF & aaagg & ccttt & agggg & $\operatorname{cccct}$ \\
\hline YAL062w & 5 & 1 & - & - \\
\hline YBL064c & 2 & - & 1 & - \\
\hline YBR072w & - & 1 & - & 3 \\
\hline YBR118w & - & - & - & - \\
\hline YBR151w & 1 & 2 & - & - \\
\hline YCL064c & 2 & - & - & - \\
\hline YCL040w & 3 & 1 & 2 & 1 \\
\hline YDL244w & - & 2 & - & - \\
\hline YDR049w & 1 & 3 & - & - \\
\hline YDR171w & - & - & 3 & - \\
\hline YDR244w & 1 & 3 & - & - \\
\hline YDR382w & 1 & - & - & - \\
\hline YER021w & 2 & 2 & 1 & 1 \\
\hline YER150w & - & 1 & - & 3 \\
\hline YFL058w & 1 & 1 & 1 & 一 \\
\hline YFL031w & 1 & 1 & - & - \\
\hline YFL014w & 6 & - & 3 & 2 \\
\hline YGL048c & - & 2 & - & - \\
\hline YGR136w & 3 & 1 & - & 1 \\
\hline YGR192c & 1 & - & 1 & 1 \\
\hline YIL022w & - & 1 & - & - \\
\hline YJL052w & 1 & 1 & 2 & - \\
\hline YJR009c & 1 & - & - & - \\
\hline YKL142w & 5 & - & 1 & - \\
\hline YKL109w & 2 & 3 & - & - \\
\hline YKL103c & - & 1 & 1 & 1 \\
\hline YKL060c & - & 1 & - & - \\
\hline YKL038w & - & 3 & - & - \\
\hline YKR011c & 3 & 2 & - & 1 \\
\hline YKR046c & 1 & - & - & - \\
\hline YKR097w & 2 & 2 & - & - \\
\hline YLR249w & 1 & 1 & - & 1 \\
\hline YLR324w & 1 & 2 & - & - \\
\hline YLR377c & - & - & - & - \\
\hline YMR267w & 4 & 1 & - & - \\
\hline YMR297w & - & 2 & 2 & - \\
\hline YMR302c & - & - & - & - \\
\hline YNL332w & 1 & 1 & 1 & - \\
\hline YNL160w & 3 & 1 & 1 & - \\
\hline YNL055c & - & 1 & - & 1 \\
\hline YNR034w & 1 & - & 1 & - \\
\hline YOL052c-a & 2 & 2 & - & 4 \\
\hline YOR008c & 2 & 1 & 1 & - \\
\hline YPL061w & 1 & - & 1 & - \\
\hline YPL048w & - & - & - & - \\
\hline YPR032w & 2 & - & 1 & - \\
\hline
\end{tabular}

The occurrence of each of the penta-nucleotides listed was scored in the $500 \mathrm{bp}$-long regions upstream of the genes hyper-expressed in the post-diauxic phase. The occurrence of the listed penta-nucleotide in the strand whose sequence was identical to that of the corresponding mRNA was scored.

the sequence upstream of almost all of the stress-responsive genes on chromosome $\mathrm{VI}$ when analyzed in detail (Masuda-Kasai, Y. and Isono, K., manuscript in preparation). We are currently investigating the effect of deleting these and other putative cis-elements from the sequence upstream of the stationary phase-re- sponsive genes on their transcriptional activity.

\section{A concluding remark}

In this paper, we have identified and partly characterized 42 genes of $S$. cerevisiae that are hyper-expressed in cells in the post-diauxic phase. Our results suggest that genes involved in various metabolic activities appear to be involved in a cell's entry into the stationary phase. Of the 42 genes analyzed, 11 were newly identified in this work as active genes which are transcribed differently in different growth phases. Some of them were further analyzed for whether or not they could be deleted by examining the growth of cells in which the genes in question had been disrupted. Furthermore, the transcriptional activity of some of the genes hyperactive in the post-diauxic phase was investigated under other stress conditions. The genes were categorized into three groups which behaved differently in response to different growth conditions. As described earlier, the amount of mRNA detected by Northern hybridization does not necessarily reflect the transcriptional activity of the corresponding gene. Nevertheless, in almost all cases analyzed, Northern hybridization data seemed to be a good indicator of the genes' transcriptional activity, since the activity measured via a reporter fused to them corresponded to the Northern hybridization data very well (not shown). It will be interesting to analyze further the effect(s) of deletion of one or more of these genes simultaneously.

We are very grateful to Drs. S. Isono and M. Kitakawa for valuable suggestions and comments on the manuscript.

\section{References}

Ausubel, F. M., Brenf, R., Kingston, R. E., Moore, D. D., Seidman, J. G., Smith, J. A., and Struhl, K. (1996) Current Protocols in Molecular Biology, Vol. 1, John Wiley and Sons, Inc., New York.

Choder, M. (1991) A general topoisomerase I-dependent transcriptional repression in the stationary phase in yeast. Genes Dev., 5, 2315-2326.

Craig, E. A., Gambill, B. D., and Nelson, J. (1993) Heat shock proteins: Molecular chaperones of protein biogenesis. Microbiol. Rev., 57, 402-414.

Dhawale, S. S. and Lane, A. C. (1993) Compilation of sequencespecific DNA-binding proteins implicated in transcriptional control in fungi. Nucl. Acids Res., 21, 5537-5546.

Drebot, M. A., Barnes, C. A., Singer, R. A., and Johnston, G. C. (1990) Genetic assessment of stationary phase for cells of the yeast Saccharomyces cerevisiae. J. Bacteriol., 172, 35843589.

Finley, D., Özaynak, E., and Varshavsky, A. (1987) The yeast polyubiquitin gene is essential for resistance to high temperatures, starvation, and other stresses. Cell, 48, 1035-1046.

Fondrat, C. and Kalogeropoulos, A. (1994) Approaching the function of new genes by detection of their potential upstream activation sequences in Saccharomyces cerevisiae: Application to chromosome III. Curr. Genet., 25, 396-406.

Ireland, L. S., Johnston, G. C., Drebot, M. A., Dhillon, N., DeMag- 
gio, A. J., Hoekstra, M. F., and Singer, R. A. (1994) A member of a novel family of yeast ' $\mathrm{Zn}$-finger' proteins mediates the transition from stationary phase to cell proliferation. EMBO J., 13, 3812-3821.

Kobayashi, N. and McEntee, K. (1990) Evidence for a heat shock transcription factor-independent mechanism for heat shock induction of transcription in Saccharomyces cerevisiae. Proc. Natl. Acad. Sci. U.S.A., 87, 6550-6554.

Kobayashi, N. and McEntee, K. (1993) Identification of cis and trans components of a novel heat shock stress regulatory pathway in Saccharomyces cerevisiae. Mol. Cell Biol., 13, 248-256.

Kolter, R., Siegele, D. A., and Tormo, A. (1993) The stationary phase of the bacterial life cycle. Annu. Rev. Microbiol., 47, 855-874.

Mager, W. H. and Ferreira, P. M. (1993) Stress response of yeast. Biochem. J., 290, 1-13.

Marchler, G., Schüller, C., Adam, G., and Ruis, H. (1993) A Saccharomyces cerevisiae UAS element controlled by protein kinase $A$ activates transcription in response to a variety of stress conditions. EMBO J., 12, 1997-2003.

McAlister, L. and Holland, M. J. (1985) Differential expression of the three yeast glyceraldehyde-3-phosphate dehydrogenase genes. J. Biol. Chem., 260, 15019-15027.

Piñon, R. (1978) Folded chromosomes in non-cycling yeast cells. Evidence for a characteristic g0 form. Chromosoma, 67, 263274.

Ruis, H. and Schüller, C. (1995) Stress signalling in yeast. BioEssays, 17, 959-965.

Sambrook, J., Fritsch, E. F., and Maniatis, T. (1989) Molecular Cloning: A Laboratory Manual, Cold Spring Harbor Laboratory, Cold Spring Harbor, New York.

Schmitt, A. P. and McEntee, K. (1996) Msn2p, a zinc finger DNAbinding protein, is the transcriptional activator of the multistress response in Saccharomyces cerevisiae. Proc. Natl. Acad. Sci. U.S.A., 93, 5777-5782.
Seifert, H. S., Chen, E. Y., So, M., and Heffron, F. (1986) Shuttle mutagenesis: A method of transposon mutagenesis for Saccharomyces cerevisiae. Proc. Natl. Acad. Sci. U.S.A., 83, 735739.

Sprague, G. F., Jr., Jensen, R., and Herskowitz, I. (1983) Control of yeast cell type by the mating type locus: Positive regulation of the $\alpha$-specific STE3 gene by the MAT $\alpha-1$ product. Cell, 32, 409-415.

Valentin, E., Herrero, E., Rico, H., Miragall, F., and Sentandreu, R. (1987) Cell wall mannoproteins during the population growth phases in Saccharomyces cerevisiae. Arch. Microbiol., 148, 88-94.

Vallari, R. C., Cook, W. J., Audino, D. C., Morgan, M. J., Jensen, D. E., Laudano, A. P., and Denis, C. L. (1992) Glucose repression of the yeast $A D H 2$ gene occurs through multiple mechanisms, including control of the protein synthesis of its transcriptional activator, ADR1. Mol. Cell. Biol.,12, 1663-1673.

Weiner, L. and Model, P. (1994) Role of an Escherichia coli stressresponse operon in stationary-phase survival. Proc. Natl. Acad. Sci. U.S.A., 91, 2191-2195.

Werner-Washburne, M., Becker, J., Kosic-Smithers, J., and Craig, E. A. (1989) Yeast Hsp70 RNA levels vary in response to the physiological status of the cell. J. Bacteriol., 171, 2680-2688.

Werner-Washburne, M., Braun, E. L., Crawford, M. E., and Peck, V. M. (1996) Stationary phase in the Saccharomyces cerevisiae. Mol. Microbiol., 19, 1159-1166.

Werner-Washburne, M., Braun, E., Johnston, G. C., and Singer, R. A. (1993) Stationary phase in yeast Saccharomyces cerevisiae. Microbiol. Rev., 57, 383-401.

Wieser, R., Adam, G., Wagner, A., Schüller, C., Marchler, G., and Ruis, H. (1991) Heat shock factor-independent heat control of transcription of the CTT1 gene encoding the cytosolic catalase T of Saccharomyces cerevisiae. J. Biol. Chem., 266, 12406-12411. 